\title{
An adaptive higher order finite element model and modal energy for the vibration of a travelling string
}

\author{
Enwei Chen ${ }^{\mathrm{a}}$, Mengbo $\mathrm{Li}^{\mathrm{a}}$, Neil Ferguson ${ }^{\mathrm{b}}$, Yimin $\mathrm{Lu}^{\mathrm{a}}$ \\ a School of Mechanical Engineering, Hefei University of Technology, China \\ ${ }^{\mathrm{b}}$ Institute of Sound and Vibration Research, University of Southampton, England, UK \\ Corresponding Author: Enwei Chen, School of Mechanical Engineering, Hefei University of Technology, \\ 230009, Hefei, China.Email:cew723@163.com
}

\begin{abstract}
A nonlinear equation describing the transverse vibration of an axially travelling string with constant and time-varying length is obtained by developing a new finite element model described by quadratic shape functions. A novel nonlinear coordinate transform is introduced with regard to its nonlinear terms. Subsequently, a new hybrid Newmark-Beta / time varying degree of freedom method, which can adjust the element number automatically according to the change of string length, is proposed to improve accuracy. The proposed method as well as normal numerical methods are compared with an analytical solution. Results show that the proposed method is in good agreement with the Newmark-Beta method for the case of small variations in string length, whilst superior in accuracy to the latter for the case of large variations. Complex mode theory is adopted firstly to obtain the modal components as well as the modal energy for a travelling string. A phenomenon is observed where the free vibration energy leaks from one mode to the others in a travelling string. The higher the speed of translation and the modal order, the more energy that is leaked into the modes close to the initially excited mode.
\end{abstract}

Keywords: adaptive DOF, higher order, travelling string, complex mode, energy transfer

\section{Introduction}

Many engineering devices which are long and narrow enough involve the transverse vibration of string-like axially moving systems. An axially moving string is a typical model widely used to represent a conveyor belt (Suweken and Van Horssen, 2003), elevator cables (Zhu and Xu, 2003), paper sheet (Benson and Stolte, 1992), pipe conveying fluid (Kuiper and Metrikine, 2004) and mine hoisting cables (Kaczmarczyk and Ostachowicz, 2003), etc. Many researchers have intensively investigated these problems over the last few decades. Typically, Hamilton's principle has been used to derive the equations of motion for a travelling string, including the continuous model represented by a partial differential equation (Carrier, 1945; Archibald and Emslie, 1958) and finite element model producing ordinary differential equations (Yao et al., 1999; Fung et al., 
1998). The classical partial differential equation for a moving string was first introduced by Archibald (1958). Later, Fung and Yao (1998, 1999) developed a hybrid Laplace transform / finite element method for a variable length string.

Approximate and analytical solutions for the moving string with both constant and variable lengths have been derived. As for the continuous model, one can obtain its approximate response by using the Galerkin discrete method (Zhang, 2008; Chen et al., 2009) or obtain its analytical solution to some initial conditions by using a wave superposition method (Chen et al., 2017). Sack (1954) gave an analytical solution for a constant length string with constant travelling speed. Kotera (1978) introduced a new coordinate transformation technology for a variable length system, which map the time-variable domain into a time-invariant one. The equation after applying coordinate transformation is not a system with time-variable coefficients and can be solved by Lapalce transformation. For the Galerkin method, it is difficult to choose a truncation order and a trial function properly, which leads to low efficiency and accuracy. Also, the wave superposition method (Chen et al., 2017) can obtain exact results of a string only for free vibration. As for the discrete model, Newmark-Beta and Runge-Kutta methods were applied to obtain approximate solutions for variable length string with fixed element number. Matsuzaki et al. (1995) compared results from FEM simulations with experimental data. Al-Bedoor, Khulief (1997) and C-M Yao et al. (1998) used FEM with variable stiffness to analyze a variable length string with fixed element number. Chen and Ferguson (2014) built a string model with either constant or variable length using a FEM model with linear shape functions. It is obvious that a fixed element number is not suitable for a string with a large variation in length due to poor accuracy. The scientific novelty of this paper lies in four following aspects. Firstly, a quadratic shape function instead of linear one is chosen for the elements to establish the string model. Secondly, a new nonlinear coordinate transform method is used to transform the nonlinear term in the system equation from local coordinate to global coordinate. Third, a time-varying degrees of freedom (TV-DOF) technique is developed to vary the number of element automatically according to the change of string length, so as to improve the accuracy. Finally, the complex mode theory is adopted to obtain the modal components as well as the modal energy for a travelling string so as to analyze the characteristics of energy transfer between modes.

The rest parts are organized as follows. The derivation of a travelling string model is presented in section 2. In section 3, the proposed solution scheme together with TV-DOF technique is introduced. Section 4 includes the numerical simulation. In section 5 , the modal energy for a travelling string are presented and the relationship governing the energy fluctuation is given. Finally, the conclusions are drawn in section 6.

\section{Derivation}

2.1 Discretisation of the governing equation for a travelling string 


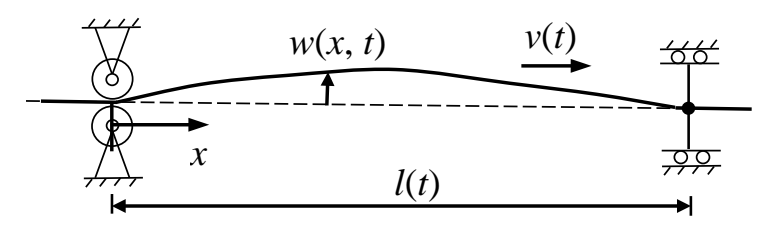

Fig.1. Travelling uniform string system with time varying length

The model in Fig. 1 is a uniform string with length $l(t)$ and a constant axial speed $v . w(x, t)$ is the transverse displacement of a point in the string in position $x$ and at time $t$ and $\dot{x}$ is the axial velocity at that point. In this paper, the length of string can be constant, i.e. $\dot{x}=v, \dot{l}(t)=0$ or changes linearly and the change rate of the string length is equal to the travelling speed of string, i.e. $\dot{x}=\dot{l}(t)=v$.

The Lagrangian function $L$ defined as follows is used to obtain the equation of motion.

$$
L=T-V
$$

Here, $T$ and $V$ are the kinetic and potential energy of the string, respectively. The expressions for $T$ and $V$ are given as follows (Yao et al., 1999)

$$
\begin{gathered}
T=\frac{1}{2} \int_{0}^{l(t)} \rho\left(w_{t}^{2}+2 w_{t} w_{x} \dot{x}+w_{x}{ }^{2} \dot{x}^{2}\right) \mathrm{d} x \\
V=\frac{1}{2} \int_{0}^{l(t)} P w_{x}{ }^{2} \mathrm{~d} x+\frac{1}{8} \int_{0}^{l(t)} E A w_{x}{ }^{4} \mathrm{~d} x
\end{gathered}
$$

where $w_{x}=\frac{\partial w}{\partial x}$ and $w_{t}=\frac{\partial w}{\partial t} . A$ is the cross-sectional area, $\rho$ is the line density, $E$ is the Young's modulus of elasticity, $P$ is the applied static tension. Using the FEM method, the displacement in element $j$ is expressed by

$$
w_{j}(x, t)=\mathbf{N}_{j}(x, l(t)) \mathbf{q}_{j}(t)
$$

where, $\mathbf{N}_{j}(x, l(t))$ is the shape function. Here a quadratic shape function is chosen to improve accuracy in contrast to a linear shape function as shown in Fig.2.

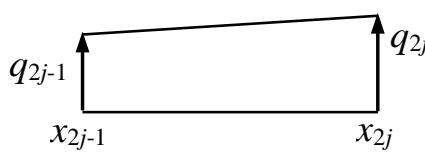

(a)

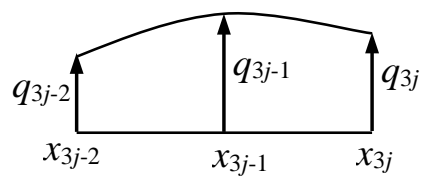

(b)

Fig.2. The linear (in (a)) and the quadratic (in (b)) shape functions are used for the $j^{\text {th }}$ element.

The detailed expression for $\mathbf{N}_{j}(x, l(t))$ is given as follows and the derivation for the quadratic 
shape function is shown in Appendix 2.

$$
\mathbf{N}_{j}=\left[-\frac{(j l(t)-n x)(l(t)-2 j l(t)+2 n x)}{l(t)^{2}}, \frac{4(j l(t)-n x)(l(t)-j l(t)+n x)}{l(t)^{2}}, \frac{(l(t)-j l(t)+n x)(l(t)-2 j l(t)+2 n x)}{l(t)^{2}}\right]
$$

The vector $\mathbf{q}_{j}$ comprises the displacements of the nodes 3j-2, 3j-1 and $3 j$ in the $j$ element shown in Eq.(6), $j=1,2, \ldots, n$ and $n$ is the element number.

$$
\mathbf{q}_{j}=\left[q_{3 j-2}(t), q_{3 j-1}(t), q_{3 j}(t)\right]^{\mathrm{T}}
$$

Table 1. Comparison of approximate frequencies with exact values for a fixed-fixed string

\begin{tabular}{cccccccc}
\hline \multirow{2}{*}{ Mode } & Exact & \multicolumn{5}{c}{ Approximate Frequencies (Hz) } \\
\cline { 3 - 8 } & Frequency & \multicolumn{3}{c}{ Linear element } & \multicolumn{3}{c}{ Quadratic element } \\
\cline { 3 - 8 } & $(\mathrm{Hz})$ & $n=3$ & $n=4$ & $n=5$ & $n=3$ & $n=4$ & $n=5$ \\
\hline 1 & 1.581 & 1.654 & 1.622 & 1.607 & 1.582 & 1.582 & 1.581 \\
2 & 3.162 & 3.698 & 3.487 & 3.372 & 3.197 & 3.174 & 3.167 \\
\hline
\end{tabular}

Increasing the number of nodes in an element can be used to improve the calculation precision for the FEM method (Petyt, 2010). The first two natural frequencies of a non-translating fixed-fixed string obtained using quadratic and linear elements are compared with the exact frequencies in Table 1. Parameters of the string are shown in Table 2. Better accuracy is obtained using the same number quadratic elements compared to the linear ones.

The element length is assumed initially to be equal. Substituting Eq.(4) into (3) and (2), the Lagrangian function $L$ in the $j^{\text {th }}$ element is

$$
\begin{aligned}
& L_{j}=T_{j}-V_{j} \\
& \quad=\frac{1}{2} \mathbf{q}_{j}{ }^{\mathrm{T}} \mathbf{k}_{j 1} \mathbf{q}_{j}+\mathbf{q}_{j}{ }^{\mathrm{T}} \mathbf{k}_{j 2} \mathbf{q}_{j}+\frac{1}{2} \mathbf{q}_{j}{ }^{\mathrm{T}} \mathbf{k}_{j 3} q_{j}+\mathbf{q}_{j}{ }^{\mathrm{T}} \mathbf{C}_{j 1} \dot{\mathbf{q}}_{j}+\dot{\mathbf{q}}_{j}{ }^{\mathrm{T}} \mathbf{C}_{j 2} \mathbf{q}_{j}+\frac{1}{2} \dot{\mathbf{q}}_{j}{ }^{\mathrm{T}} \mathbf{m}_{j} \dot{\mathbf{q}}_{j}-\mathbf{S}_{j}\left(q_{3 j-2}, q_{3 j-1}, q_{3 j}\right)
\end{aligned}
$$

$\mathbf{S}_{j}$ is given by

$$
\mathbf{S}_{j}\left(q_{3 j-2}, q_{3 j-1}, q_{3 j}\right)=\frac{1}{8} E A \int_{x_{2 j-1}}^{x_{2 j+1}} \mathbf{q}_{j}^{\mathrm{T}} \mathbf{N}_{x}^{\mathrm{T}} \mathbf{N}_{x} \mathbf{q}_{j} \mathbf{q}_{j}^{\mathrm{T}} \mathbf{N}_{x}^{\mathrm{T}} \mathbf{N}_{x} \mathbf{q}_{j} \mathrm{~d} x
$$

The detailed expressions for $\mathbf{k}_{j 1}, \mathbf{k}_{j 2}, \mathbf{k}_{j 3}, \mathbf{c}_{j 1}, \mathbf{c}_{j 2}, \mathbf{m}_{j}$ and $\hat{\mathbf{S}}_{j}\left(q_{3 j-2}, q_{3 j-1}, q_{3 j}\right)$ are shown in Appendix 1. Using the Lagrangian function, the equations of motion are given by

$$
(\mathrm{d} / \mathrm{d} t) \partial L_{j} / \partial \dot{\mathbf{q}}_{j}{ }^{\mathrm{T}}-\partial L_{j} / \partial \mathbf{q}_{j}{ }^{\mathrm{T}}=\mathbf{f}_{j}
$$

where $\mathbf{f}_{j}$ is the nonconservative force acting on the $j^{\text {th }}$ element. So the governing equation for each 
element is

$$
\mathbf{m}_{j} \ddot{\mathbf{q}}_{j}+\mathbf{c}_{j} \dot{\mathbf{q}}_{j}+\mathbf{k}_{j} \mathbf{q}_{j}+\hat{\mathbf{S}}_{j}\left(q_{3 j-2}, q_{3 j-1}, q_{3 j}\right)=\mathbf{f}_{j}
$$

where,

$$
\begin{gathered}
\mathbf{k}_{j}=\dot{\mathbf{c}}_{j 1}{ }^{\mathrm{T}}+\dot{\mathbf{C}}_{j 2}-\mathbf{k}_{j 1}-2 \mathbf{k}_{j 2}-\mathbf{k}_{j 3} \\
\mathbf{c}_{j}=\mathbf{c}_{j 1}{ }^{\mathrm{T}}+\mathbf{C}_{j 2}+\mathbf{m}_{j}-\mathbf{c}_{j 1}-\mathbf{c}_{j 2}{ }^{\mathrm{T}}
\end{gathered}
$$

The governing equation for the travelling string in the local coordinate system is

$$
\mathbf{M}_{\mathrm{L}}(t) \ddot{\mathbf{q}}+\mathbf{C}_{\mathrm{L}}(t) \dot{\mathbf{q}}+\mathbf{K}_{\mathrm{L}}(t) \mathbf{q}+\mathbf{N}_{\mathrm{L}}(t)=\mathbf{F}
$$

where, $\mathbf{q}$ is the lateral displacement vector of each node and $\mathbf{M}_{\mathrm{L}}(t), \mathbf{C}_{\mathrm{L}}(t), \mathbf{K}_{\mathrm{L}}(t), \mathbf{N}_{\mathrm{L}}(t)$ are time dependent matrices, the detailed expressions of which are shown in Appendix 1.

\subsection{Nonlinear coordinate transformation}

The relationship between $\mathbf{q}$ in local coordinate and $\mathbf{Q}$ in global coordinate is shown in Fig. 3.

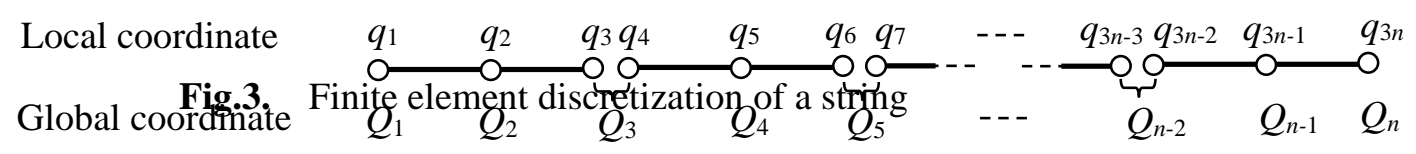

In order to obtain the response for each node, a transformation from the local ${ }^{2}$ - coordinate $\mathbf{q}^{n}$ in Eq.(13) into a global coordinate $\mathbf{Q}$ is required. However, a linear transformation is unsuitable due to the nonlinear term $N_{\mathrm{L}}(t)$ in Eq.(13). Here, a nonlinear transformation is given. Firstly, a linear transformation is applied to the first three terms of Eq.(13)

$$
\mathbf{q}=\mathbf{B Q}
$$

where $\mathbf{B}$ is constructed according to the relationship of $\mathbf{Q}$ and $\mathbf{q}$ in Fig. 3, the detailed expressions of which is shown in Appendix 1. Then one has

$$
\mathbf{M}_{\mathrm{L}}(t) \mathbf{B} \ddot{\mathbf{Q}}+\mathbf{C}_{\mathrm{L}}(t) \mathbf{B} \dot{\mathbf{Q}}+\mathbf{K}_{\mathrm{L}}(t) \mathbf{B} \mathbf{Q}+\mathbf{N}_{\mathrm{L}}(t)=\mathbf{F}_{\mathrm{L}}
$$

Left multiply by $\mathbf{B}^{\mathrm{T}}$, Eq.(15) becomes

$$
\mathbf{M}(t) \ddot{\mathbf{Q}}+\mathbf{C}(t) \dot{\mathbf{Q}}+\mathbf{K}(t) \mathbf{Q}+\mathbf{N}(t)=\mathbf{F}(t)
$$


where, $\mathbf{M}(t), \mathbf{C}(t), \mathbf{K}(t), \mathbf{N}(t)$ and $\mathbf{F}(t)$ are the global matrices given by

$$
\left\{\begin{aligned}
\mathbf{M}(t) & =\mathbf{B}^{\mathrm{T}} \mathbf{M}_{\mathbf{L}}(t) \mathbf{B} \\
\mathbf{C}(t) & =\mathbf{B}^{\mathrm{T}} \mathbf{C}_{\mathbf{L}}(t) \mathbf{B} \\
\mathbf{K}(t) & =\mathbf{B}^{\mathrm{T}} \mathbf{K}_{\mathbf{L}}(t) \mathbf{B} \\
\mathbf{N}(t) & =\mathbf{B}^{\mathrm{T}} \mathbf{N}_{\mathbf{L}}(t) \\
\mathbf{F}(t) & =\mathbf{B}^{\mathrm{T}} \mathbf{F}_{\mathbf{L}}(t)
\end{aligned}\right.
$$

Here, $\mathbf{N}(t)$ still has the local coordinates $q_{j}(j=1,2, \ldots, 3 n)$, i.e.

$$
\mathbf{N}(t)=\mathbf{B}^{\mathrm{T}} \mathbf{N}_{\mathrm{L}}(t)=\mathbf{B}^{\mathrm{T}}\left[\begin{array}{c}
\hat{\mathbf{S}}_{1} \\
\hat{\mathbf{S}}_{2} \\
\vdots \\
\hat{\mathbf{S}}_{3 n}
\end{array}\right]
$$

where $\hat{\mathbf{S}}_{i}$ is a function of $q_{j}, \quad(i=1, \cdots, 3 n ; j=1, \cdots, 3 n)$ which is shown in Appendix 1 . So $\mathbf{N}(t)$ needs further nonlinear coordinate transformation so as to be represented only by global coordinates. From Eq. (14), one has the nonlinear relationship between the local coordinate $\mathbf{q}$ and global coordinate $\mathbf{Q}$.

$$
\begin{aligned}
& q_{i}= \begin{cases}Q_{2 k-1} & i=3 k-2 \\
Q_{2 k} & i=3 k-1 \\
Q_{2 k+1} & i=3 k\end{cases} \\
& (k=1,2, \cdots, n)
\end{aligned}
$$

The nonlinear coordinate transformation for $\mathbf{N}(t)$ is completed by replacing all the local coordinate value $q_{i}$ with global coordinate value $Q$ according to Eq.(19).

\section{Solution schemes}

For the TV-DOF case, the Newmark-Beta method is no longer applicable due to the changing of element number. Here, a new hybrid Newmark-Beta/TV-DOF method is presented. The process of the method from time step $i$ to $i+1$ is introduced.

Firstly, the variation of element number $\Delta n_{i+1}$ at time step $i+1$ is obtained by

$$
\Delta n_{i+1}=\operatorname{round}\left(\left[l\left(t_{i+1}\right)-l\left(t_{i}\right)\right] / \mathrm{d} l\right)
$$

where $\mathrm{d} l$ is the element length which is set as constant and round $(\cdot)$ is defined as a function which returns an integer closest to the independent variable. Note that the dimension of $\mathbf{Q}, \dot{\mathbf{Q}}$ and $\ddot{\mathbf{Q}}$ is $2 n_{i}+1$ at time step $i$ and is $2 n_{i+1}+1$ at time step $i+1$. Recalling the Newmark-Beta method, $\mathbf{Q}_{i+1}, \dot{\mathbf{Q}}_{i+1}$ and $\ddot{\mathbf{Q}}_{i+1}$ are obtained by iterating over the values of $\mathbf{Q}_{i}, \dot{\mathbf{Q}}_{i}$ and $\ddot{\mathbf{Q}}_{i}$. If $n_{i+1} \neq n_{i}$ due to a variation of length, the Newmark-Beta method is not applicable. To solve it, a cubic spline 
interpolation(Li, 2008) is applied to $\mathbf{Q}_{i}, \dot{\mathbf{Q}}_{i}$ and $\ddot{\mathbf{Q}}_{i}$.

$$
\begin{array}{r}
\mathbf{I} \mathbf{Q}_{i}=\operatorname{CSI}\left(x_{i}, \mathbf{Q}_{i}, 2 n_{i+1}+1\right) \\
\mathbf{I} \dot{\mathbf{Q}}_{i}=\operatorname{CSI}\left(x_{i}, \dot{\mathbf{Q}}_{i}, 2 n_{i+1}+1\right) \\
\mathbf{I} \ddot{\mathbf{Q}}_{i}=\operatorname{CSI}\left(x_{i}, \ddot{\mathbf{Q}}_{i}, 2 n_{i+1}+1\right)
\end{array}
$$

where $\operatorname{CSI}(x, \mathbf{Q}, n)$ is a function which returns an $n$-dimension result after cubic spline interpolation for vector $\mathbf{Q}$ over the relevant range of $\boldsymbol{x}$. So $\mathbf{I} \mathbf{Q}_{\mathrm{i}}, \mathbf{I} \dot{\mathbf{Q}}_{i}$ and $\mathbf{I} \ddot{\mathbf{Q}}_{i}$ have the same dimension as $\mathbf{Q}_{i+1}, \dot{\mathbf{Q}}_{i+1}$ and $\ddot{\mathbf{Q}}_{i+1}$, i.e. $2 n_{i+1}+1$. By replacing $\mathbf{Q}_{i}, \dot{\mathbf{Q}}_{i}$ and $\ddot{\mathbf{Q}}_{i}$ into $\mathbf{I} \mathbf{Q}_{\mathrm{i}}, \mathbf{I} \dot{\mathbf{Q}}_{i}$ and $\mathbf{I} \ddot{\mathbf{Q}}_{i}$, respectively, the Newmark-Beta method can be applied to the TV-length string case.

In this way, the vectors of displacement and velocity for Eq. (16) at time step $i+1$ are

$$
\begin{gathered}
\mathbf{Q}_{i+1}=\mathbf{I} \mathbf{Q}_{i}+\mathbf{I} \dot{\mathbf{Q}}_{i} \Delta t+\frac{1}{4}\left(\mathbf{I} \ddot{\mathbf{Q}}_{i}+\ddot{\mathbf{Q}}_{i+1}^{*}\right) \Delta t^{2} \\
\dot{\mathbf{Q}}_{i+1}=\mathbf{I} \dot{\mathbf{Q}}_{i}+\frac{1}{2}\left(\mathbf{I} \ddot{\mathbf{Q}}_{i}+\ddot{\mathbf{Q}}_{i+1}^{*}\right) \Delta t
\end{gathered}
$$

where, $\ddot{\mathbf{Q}}_{i+1}^{*}$ is

$\boldsymbol{\delta}$ is a $2 n+1$-dimensional small random vector. Then $\stackrel{\mathbf{Q}}{i+1}_{i}$ can be obtained

$$
\begin{gathered}
\ddot{\mathbf{Q}}_{i+1}^{*}= \begin{cases}\boldsymbol{\delta} & (i=1) \\
\ddot{I}_{i}+\boldsymbol{\delta} & (i>1)\end{cases} \\
\text { Then } \ddot{\mathbf{Q}}_{i+1} \text { can be obtained }
\end{gathered}
$$

$$
\ddot{\mathbf{Q}}_{i+1}=-\mathbf{M}_{i+1}^{-1}\left(\mathbf{C}_{i+1} \dot{\mathbf{Q}}_{i+1}+\mathbf{K}_{i+1} \mathbf{Q}_{i+1}+\mathbf{N}_{i+1}-\mathbf{B}^{\mathrm{T}} \mathbf{F}_{i+1}\right)
$$

Comparing the 2-norm of the computed $\ddot{\mathbf{Q}}_{i+1}$ with the assumed $\ddot{\mathbf{Q}}_{i+1}^{*}$, if they are equal or within a permissible difference $\varepsilon$, the process for time step $i+1$ is finished. Otherwise, let $\ddot{\mathbf{Q}}_{i+1}$ to be the assumed $\ddot{\mathbf{Q}}_{i+1}^{*}$ in the next iteration in Eqs.(24), (25) and (27), and continue to calculate $\mathbf{Q}_{i+1}$ and $\dot{\mathbf{Q}}_{i+1}$ till they are convergent. By repeating the above process for all time steps, one can obtain $\mathbf{Q}, \dot{\mathbf{Q}}$ and $\ddot{\mathbf{Q}}$ finally.

\section{Numerical simulations}

\subsection{Constant length case}

In this case, the parameters of string are given in Table2. The assumed initial conditions are

$$
\left\{\begin{array}{l}
w(x, 0)=A_{0} \sin (\pi x / l) \\
\dot{w}(x, 0)=0
\end{array}\right.
$$

The boundary conditions are

$$
w(0, t)=w(l(t), t)=0
$$

Table 2. Parameters of the string model 


\begin{tabular}{cccc}
\hline parameters & values/unit & parameters & values/unit \\
\hline$l$ & $3 \mathrm{~m}$ & $N$ & 50 \\
$i(t)$ & $0 \mathrm{~m} / \mathrm{s}$ & $v$ & $\pm 0.32 c_{0}$ \\
$E A$ & $3.2 \times 10^{4} \mathrm{~N}$ & $A_{0}$ & $0.01 \mathrm{~m}$ \\
$\rho$ & $0.1 \mathrm{~kg} / \mathrm{m}$ & $n$ & 20 \\
$\Delta t$ & $0.02 \mathrm{~s}$ & $P$ & $1 \mathrm{~N}$ \\
\hline
\end{tabular}

Here the Newmark-Beta, the Runge-Kutta and the TV-state space function method(Chen and Ferguson, 2014) are used to solve Eq.(16) compared to the analytical method in (Chen et al., 2017), which gives exact results.

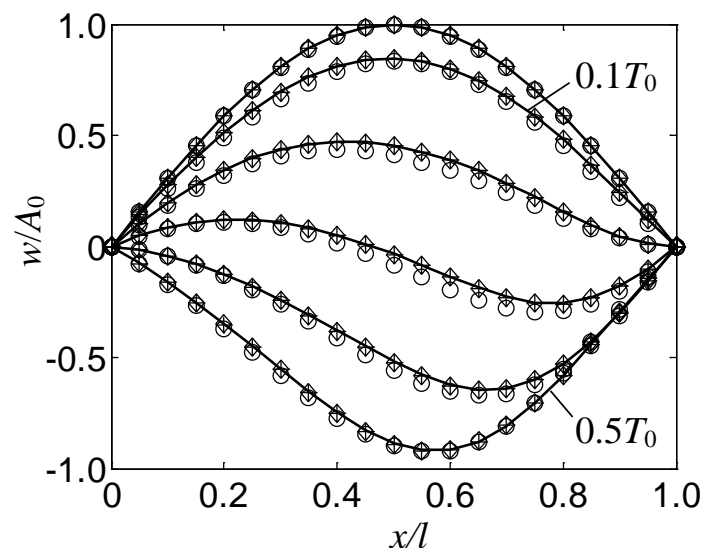

(a)

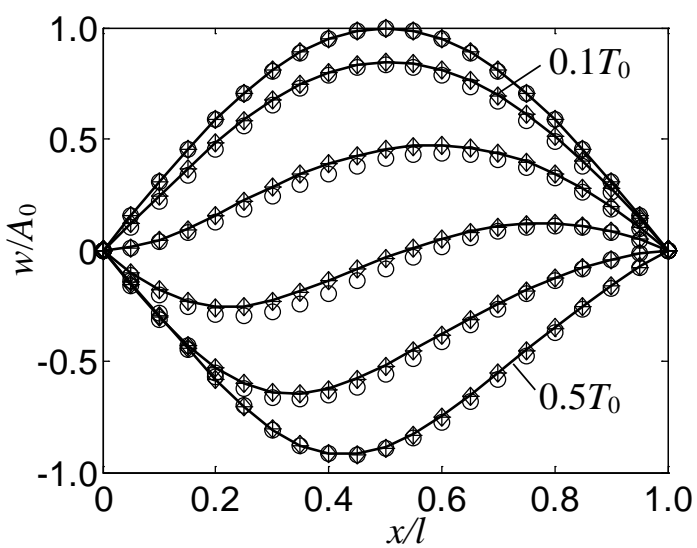

(b)

Fig.4. Comparison of the nodal displacements with opposite directions of string translation during $0.5 T_{0}$. The curves are identified using — the analytical solution, $\bigcirc \circ \bigcirc$ the Newmark-Beta, +++ the Runge-Kutta and $\diamond \diamond \diamond$ the TV- state space function methods. The speeds are $1 \mathrm{~m} / \mathrm{s}$ in (a) and $-1 \mathrm{~m} / \mathrm{s}$ in (b).

In Fig.4, the initial deflected shape of the string is in its first mode shape, while its free vibration is no longer a pure modal vibration. The six curves in each plot are at the corresponding times of $0,0.1 T_{0}, 0.2 T_{0}, 0.3 T_{0}, 0.4 T_{0}$ and $0.5 T_{0} . T_{0}$ is the minimum cycle required to return to the initial deflection shape of a string with constant length which is given by (Chen et al., 2017)

$$
T_{0}=\frac{2 c_{0} l}{c_{0}^{2}-v^{2}}
$$

where $c_{0}$ is the free wave speed in the non-translating string, i.e.

$$
c_{0}=\sqrt{P / \rho}
$$

It is observed in Fig. 6 that the results using the three numerical methods with quadratic shape functions are almost consistent with the analytical solution. 
Four cases with different translational speed, i.e. $v=0.3 c 0,0.6 c 0,0.9 c 0$ and $c 0$, are shown in Fig.5(a), (b), (c) and (d) using the Newmark-Beta method with the parameters in Table2. Here, $c_{0}=3.16 \mathrm{~m} / \mathrm{s}$. When the translational speed is high, the waves are blocked in the opposite direction of string moving. When the travelling velocity is close to $c_{0}$, divergence is produced due to the energy accumulates at some points along the length, as shown in Fig.5(d).

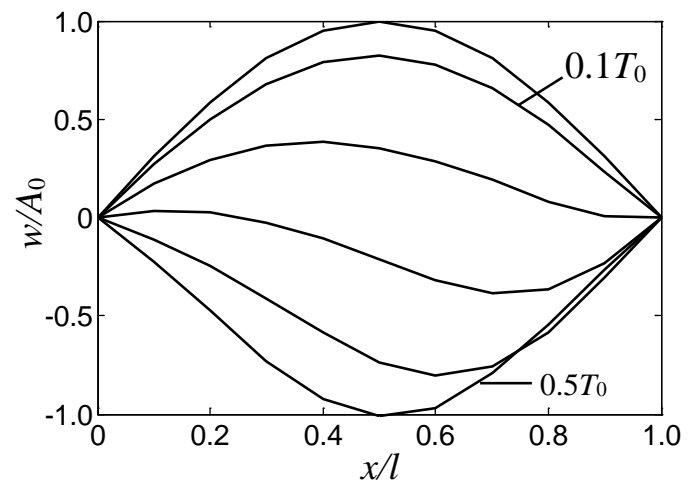

(a)

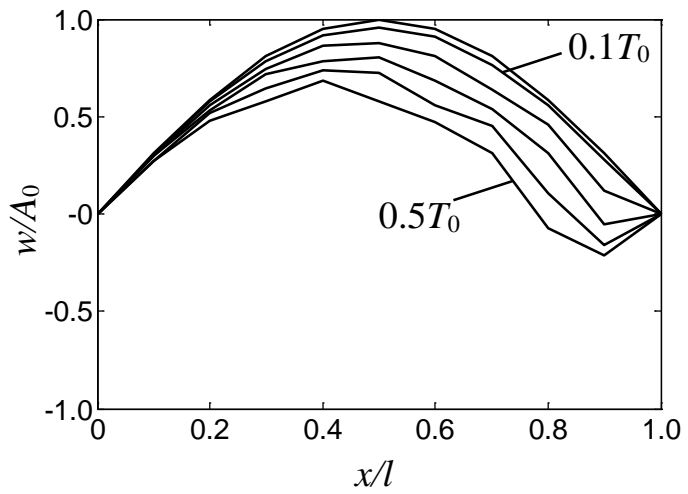

(c)

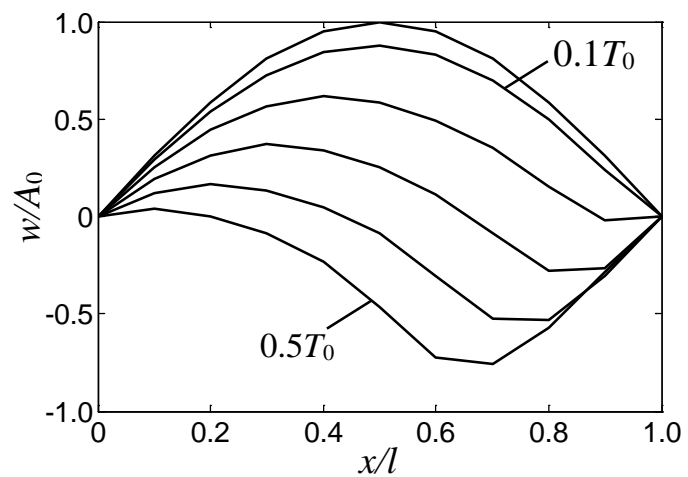

(b)

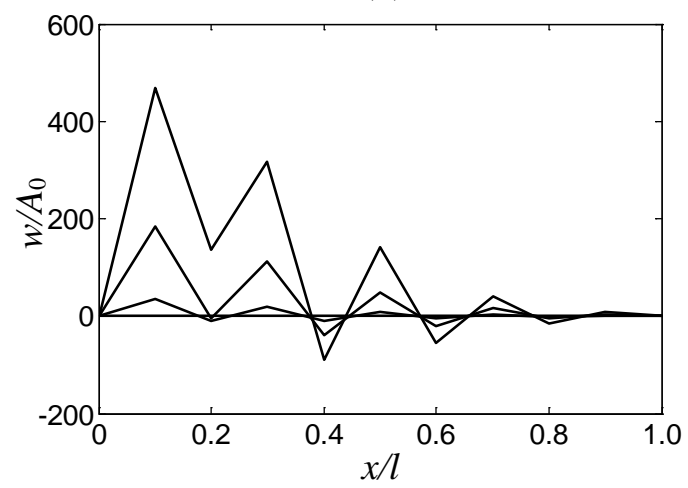

(d)

Fig.5. Displacement of a constant length string with velocities of (a) $v=0.3 c_{0}$, (b) $v=0.6 c_{0}$, (c) $v=0.9 c_{0}$ and (d) $v=c_{0}$, respectively.

In order to study the influence of nonlinear term $N(t)$ in the governing equation on vibration response, the orthogonal test method is adopted here. Because the nonlinear term $N(t)$ is affected by factors such as the product of elastic modulus $E$ and cross-sectional area of string $A$, i.e. $E A$, traveling velocity $v$ and the ratio of maximum amplitude $A_{0}$ and string length $l$, these factors are selected for orthogonal test. The values of each factor have three levels, as shown in Table 3. The test target $R$ is defined in Eq.(32) and shown in Table 4.

$$
R=\sum\left[\mathbf{Q}\left(T_{0} / 2\right)-\mathbf{Q n o}_{\mathbf{n o}}\left(T_{0} / 2\right)\right] /\left(n A_{0}\right)
$$

where, $\mathbf{Q}_{\mathbf{n o}}$ is the lateral displacement vector without the nonlinear term.

Table 3. Test factors and levels 


\begin{tabular}{ccccc}
\hline & factors & \multicolumn{3}{c}{ levels } \\
\cline { 3 - 5 } & & 1 & 2 & 3 \\
\hline A & $E A(\mathrm{~N})$ & $10^{4}$ & $3 \times 10^{4}$ & $6 \times 10^{4}$ \\
B & $v$ & $0.1 c_{0}$ & $0.3 c_{0}$ & $0.5 c_{0}$ \\
C & $A_{0} / l$ & $0.15 \%$ & $0.35 \%$ & $0.65 \%$ \\
\hline
\end{tabular}

Table 4. Orthogonal test results

\begin{tabular}{|c|c|c|c|c|}
\hline \multirow{2}{*}{ Test } & \multicolumn{3}{|c|}{ Levels of factors } & \multirow{2}{*}{$R$} \\
\hline & A & $\mathrm{B}$ & $\mathrm{C}$ & \\
\hline 1 & 1 & 1 & 1 & $0.11 \%$ \\
\hline 2 & 1 & 2 & 2 & $0.44 \%$ \\
\hline 3 & 2 & 3 & 3 & $6.00 \%$ \\
\hline 4 & 2 & 1 & 3 & $2.38 \%$ \\
\hline 5 & 3 & 2 & 2 & $2.65 \%$ \\
\hline 6 & 3 & 3 & 1 & $0.74 \%$ \\
\hline
\end{tabular}

From the results shown in Table 4, the ranges for three factors, i.e. $E A, v$ and $A_{0} / l$, are $7.83 \%$, $4.25 \%$ and $7.53 \%$, respectively after calculation. It can be seen after comparison that $E A$ is the main factor for the influence of nonlinearity and $A 0 / l$ is the second. Fig. 6 shows the comparisons of displacement responses with and without nonlinear term for the test 1 ( $R$ minimum ) and the test 3 ( $R$ maximum ).

$\underset{3}{3}$

$\frac{\mathbb{1}^{3}}{3}$

(a)

$x / l$

(b)

$x / l$

Fig.6 Comparisons of the influence of nonlinearity on displacement responses for (a) test 1 ( $R$ minimum ) and (b) test 3 ( $R$ maximum ). Key: — with nonlinear term; ${ }^{-} \mathrm{O}^{-} \mathrm{O}^{-}$- without nonlinear term.

\subsection{Time-varying length case}

In this case, the rate of change of length is $i(t)=v$. The new hybrid Newmark-Beta/TV-DOF method is used for comparison to the fixed element number technique as well as the analytical method. The parameters and the boundary conditions are identical to those in Section 4.1, except for the initial number of elements $n(0)=10$. According to the article(Chen et al., 2017), the cycle $T_{i}$ of a variable length string is defined as 


$$
T_{i}=2 l_{0} \frac{\left(c_{0}+v\right)^{i-1}}{\left(c_{0}-v\right)^{i}}, \quad(i=1,2, \cdots)
$$

Considering the continuity for the analytical method, the initial conditions are chosen as (Chen et al., 2017):

$$
\left\{\begin{aligned}
w(x, 0) & =A_{0} \sin ^{2}\left(\pi x / l_{0}\right) \\
\dot{w}(x, 0) & =0
\end{aligned}\right.
$$

where $l_{0}$ is the initial string length.

Fig. 7 shows the comparison of the responses using three methods. The length of string varies from $0.7 l_{0}$ to $1.4 l_{0}$ in Fig.7(a) to (d) during 0 to $0.5 T_{1}$. Clearly, the element number $n$ remains constant for the Newmark-Beta method, while varies from 10 to 14 in Fig.7(b) $(i(t)=$ $\left.0.32 c_{0}\right)$ and 10 to 8 in Fig.7(d) $\left(i(t)=-0.32 c_{0}\right)$ for the new hybrid method. The curves of both methods almost coincide with those of the analytical method in Fig.7(a) to (d). On the contrary, in Fig.7(e) and (f), the length of string during $T_{1}+0.5 T_{2}$ to $T_{1}+T_{2}$ increases to about $3.5 l_{0}$ and the element number increases to 37 in Fig. 7 (f) from the original one $n=10$.

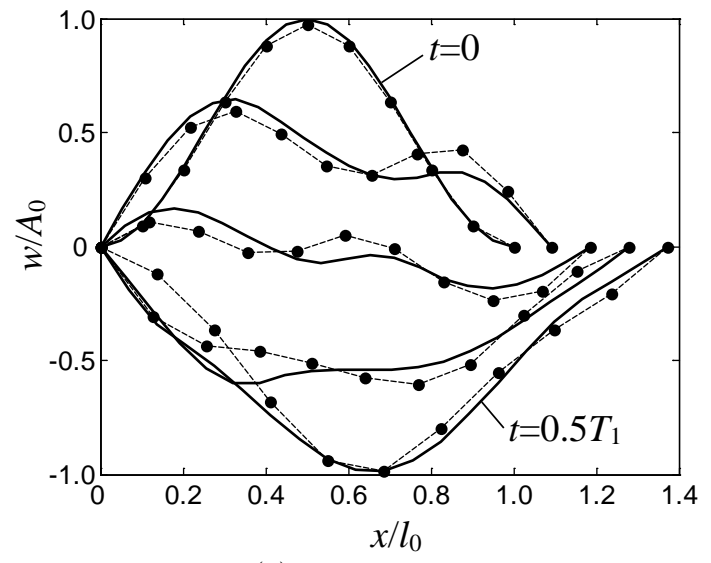

(a)

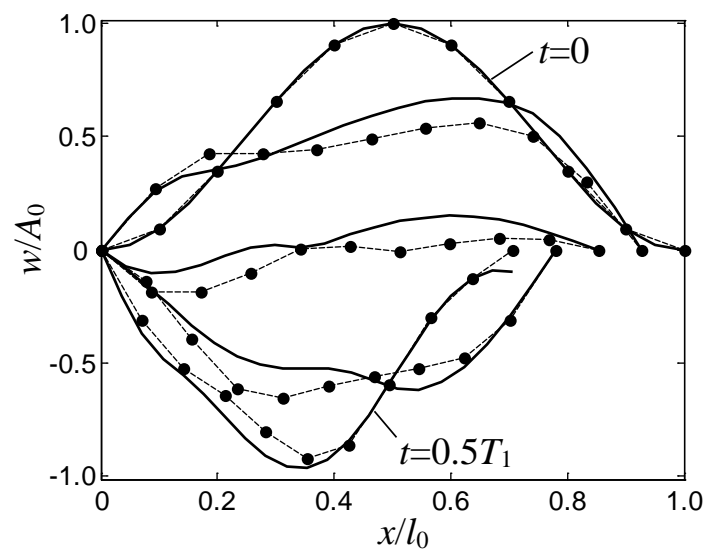

(c)

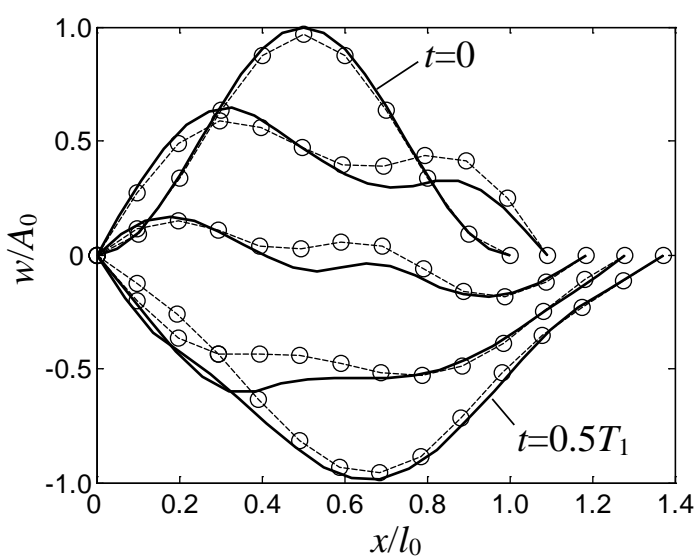

(b)

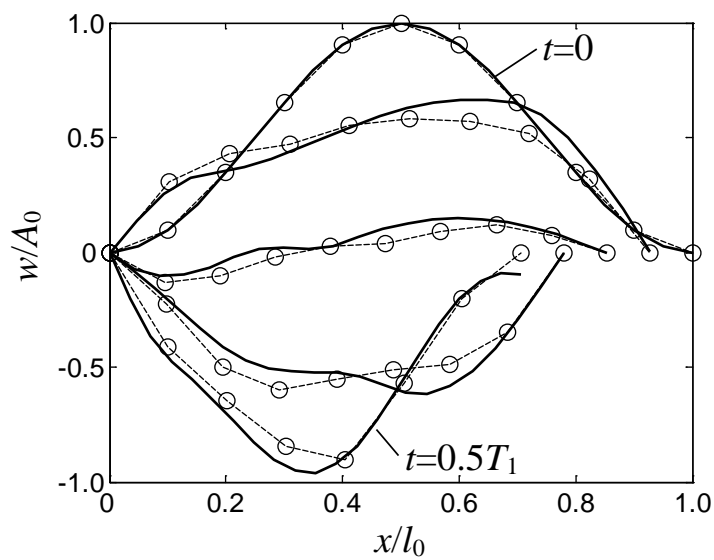

(d) 


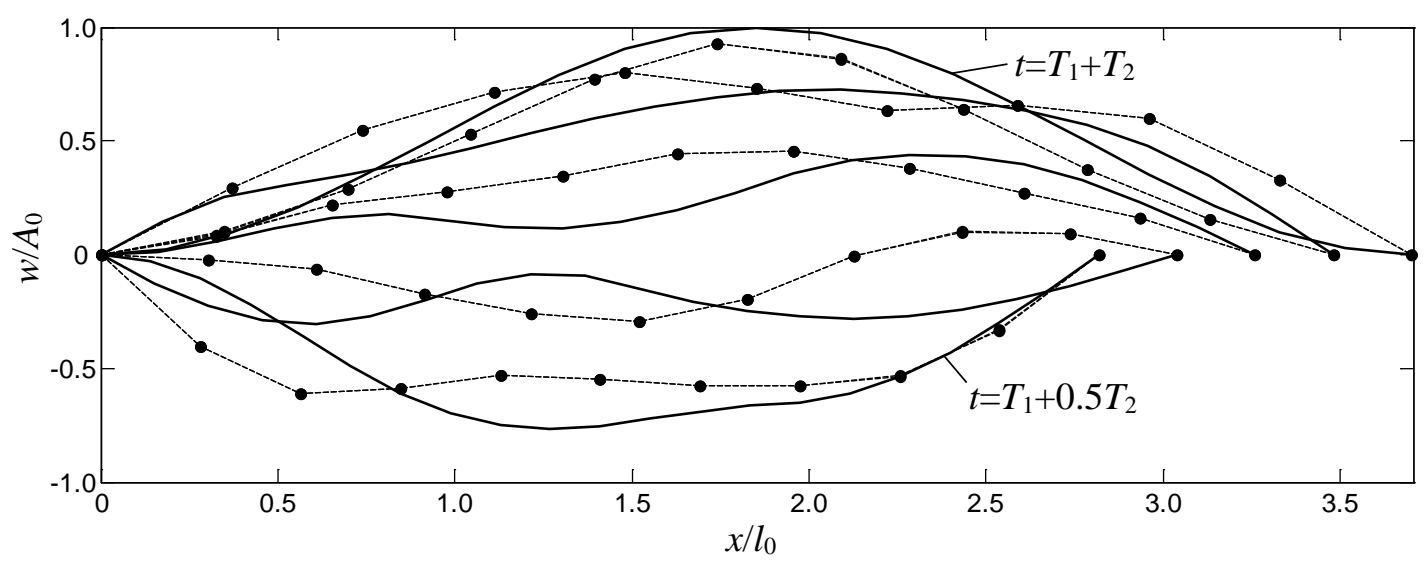

(e)

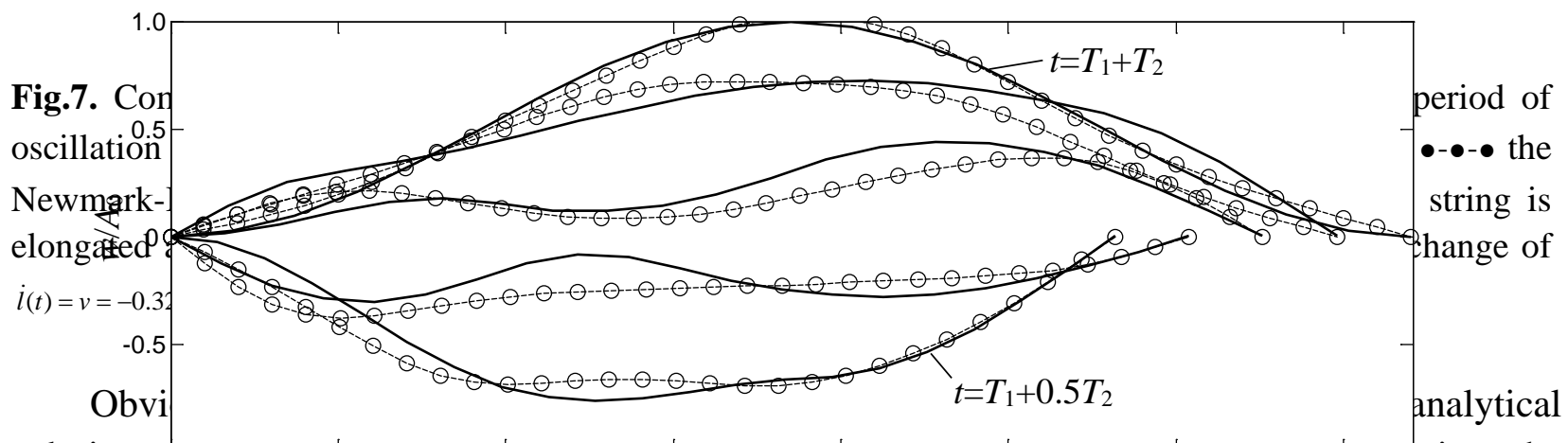

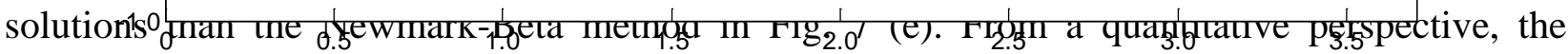
RMSE (Root-Mean-Square Errors) are 0.069 xfier the proposed method and 0.169 for the Newmark-Beta method, which are evaluated by

$$
R M S E=\sqrt{\frac{\sum_{i=1}^{n_{1}}\left[Q(i)-Q_{A}(i)\right]^{2}}{n_{1}}}
$$

where $Q$ and $Q_{A}$ are the nodal displacements for numerical and analytical methods, respectively. $n_{1}$ is the sum of node number in curve at times of $T_{1}+\frac{1}{2} T_{2}, T_{1}+\frac{5}{8} T_{2}, T_{1}+\frac{3}{4} T_{2}, T_{1}+\frac{7}{8} T_{2}$ and $T_{1}+T_{2}$, respectively.

It shows that the new hybrid method is similar to the Newmark-Beta method for the case of small variations in length while superior in accuracy for large variations in length. 


\section{Modal energy analysis}

\subsection{Modal components for a travelling string}

The free vibration for a fixed-fixed non-translating string without damping is composed of all time-invariant modes. When the initial deflected shape is in its fundamental mode shape, the mechanical energy will exist in this mode only. However, for a travelling string described in Eq.(20), a time-varying gyroscopic term exists in $\boldsymbol{C}(t)$ due to the travelling speed $v(t)$, so the modes of the string are no longer time-invariant. In this part, a time-varying mode is proposed. The characteristic matrix $\boldsymbol{\Lambda}(t)$ for Eq.(13) neglecting the nonlinear term is given as follows.

The eigenvectors matrix of $\boldsymbol{\Lambda}(t)$ is

$$
\Lambda(t)=-\left[\begin{array}{cc}
\mathbf{K}(t) & \mathbf{O} \\
\mathbf{O} & -\mathbf{M}(t)
\end{array}\right]^{-1}\left[\begin{array}{cc}
\mathbf{C}(t) & \mathbf{M}(t) \\
\mathbf{M}(t) & \mathbf{O}
\end{array}\right]
$$

$$
\Phi(t)=\left[\psi_{1}(t), \psi_{1}^{*}(t), \cdots, \psi_{n}(t), \psi_{n}{ }^{*}(t)\right]
$$

Here, $\boldsymbol{\psi}_{i}(t)$ and $\boldsymbol{\psi}_{i}^{*}(t)$ are the $i^{\text {th }}$ eigenvector and its conjugate vector of $\boldsymbol{\Lambda}(t)$, respectively.

$$
\boldsymbol{\psi}_{i}(t)=\left\{\begin{array}{c}
\boldsymbol{\varphi}_{i}(t) \\
p_{i}(t) \boldsymbol{\varphi}_{i}(t)
\end{array}\right\}
$$

$\boldsymbol{\varphi}_{i}(t)$ is the $i^{\text {th }}$ order displacement mode and $p_{i}(t)$ is the $i^{\text {th }}$ eigenvalue of $\boldsymbol{\Lambda}(t)$. The transverse displacement $\mathbf{Q}(t)$ and velocity $\dot{\mathbf{Q}}(t)$ are expressed using modal coordinate $\mathbf{Z}(t)$.

$$
\begin{gathered}
{\left[\begin{array}{c}
\mathbf{Q}(t) \\
\dot{\mathbf{Q}}(t)
\end{array}\right]=\boldsymbol{\Phi}(t) \mathbf{Z}(t)} \\
\mathbf{Z}(t)=\left[z_{1}(t), z_{1}^{*}(t), \cdots, z_{n}(t), z_{n}^{*}(t)\right]^{\mathrm{T}}
\end{gathered}
$$

So, $\mathbf{Z}(t)$ is obtained from Eq.(39)

$$
\mathbf{Z}(t)=\boldsymbol{\Phi}(t)^{-1}\left[\begin{array}{c}
\mathbf{Q}(t) \\
\dot{\mathbf{Q}}(t)
\end{array}\right]
$$

Substituting Eqs.(37), (38) and (40) into Eq.(39), one has

$$
\left[\begin{array}{c}
\mathbf{Q}(t) \\
\dot{\mathbf{Q}}(t)
\end{array}\right]=\sum_{i=1}^{n}\left\{z_{i}(t)\left[\begin{array}{c}
\boldsymbol{\varphi}_{i}(t) \\
p_{i}(t) \boldsymbol{\varphi}_{i}(t)
\end{array}\right]+z_{i}^{*}(t)\left[\begin{array}{c}
\boldsymbol{\varphi}_{i}^{*}(t) \\
p_{i}^{*}(t) \boldsymbol{\varphi}_{i}^{*}(t)
\end{array}\right]\right\}
$$

Eq.(42) shows the responses are composed of $n$ modal components, so the $i^{\text {th }}$ modal components for displacement and velocity are

$$
\mathbf{Q}_{M i}(t)=Z_{i}(t) \varphi_{i}(t)+Z_{i}^{*}(t) \boldsymbol{\varphi}_{i}^{*}(t)
$$

and 


$$
\dot{\mathbf{Q}}_{\mathrm{M} i}(t)=z_{i}(t) p_{i}(t) \varphi_{i}(t)+Z_{i}^{*}(t) p_{i}^{*}(t) \varphi_{i}^{*}(t)
$$

Eqs.(43) and (44) show the modal shape $\varphi_{i}(t)$ is a time function which changes with travelling speed $v$. With $v$ and the order of mode increasing, $\boldsymbol{\varphi}_{i}(t)$ changes more significantly compared to the stationary one. These changes at different $v$ causes the energy transferring from one mode to another although existing initially only in one mode.

\subsection{Modal energy}

Replacing the displacement $w$ in Eqs.(2) and (3) with $\mathbf{Q}_{M i}$ in Eq.(43), one has the kinetic and potential energies for the $i^{\text {th }}$ mode, respectively

$$
\begin{gathered}
T_{M i}(t)=\frac{1}{2} \int_{0}^{l(t)} \rho\left(Q_{M i t}{ }^{2}+2 Q_{M i t} Q_{M i x} \dot{x}+Q_{M i x}{ }^{2} \dot{x}^{2}\right) \mathrm{d} x \\
V_{M i}(t)=\frac{1}{2} \int_{0}^{l(t)} T_{0} Q_{M i x}{ }^{2} \mathrm{~d} x+\frac{1}{8} \int_{0}^{l(t)} E A Q_{M i x}{ }^{4} \mathrm{~d} x
\end{gathered}
$$

The total energy is

$$
E_{M i}(t)=T_{M i}(t)+V_{M i}(t)
$$

The initial conditions of the string are the first four mode shapes as follows

The energy in each mode is calculated and compared between travelling speeds of $0,0.3 c_{0}$ and $0.6 c 0$ and four initial conditions in Eq.(48). Other parameters are given in Table 2.

The non-dimensional energy $E_{M i}(t) / E_{\mathrm{T}}(t)$ in each mode is shown in Fig.8. Here $E_{\mathrm{T}}(t)$ is the total energy of the string at time $t$. In these four sub plots, the initial displacements are the lowest four mode shapes, respectively. For a fixed-fixed string without translation, all the energy exists only in its initial mode shape. However, for the travelling string under same initial conditions as above, the free vibration is no longer a pure mode and the energy is transferred from the excited mode to its adjacent modes. From the comparisons of the non-dimensional energy between the twelve cases, it shows the higher the travelling speed is, the more energy is transferred. For example, in Fig.8(b), the energy kept in the second mode, which is the excited mode, reduces from $100 \%$ to about $80 \%$ when $v$ changes from 0 to $0.3 c_{0}$. In Fig.8(d), the energy kept in the 4th mode, which is the excited mode, reduces from $100 \%$ to less than $10 \%$ while the energies in adjacent third and fourth mode increase significantly from 0 to about $50 \%$ and $30 \%$, respectively, when $v$ changes from 0 to $0.6 c 0$. At the same translational speed, the energy in higher mode is easier to transfer to others than in lower mode. For example, the first, second and third modal energy in (a), (b) and (c) reduce from $100 \%$ to $80 \%, 30 \%$ and less than $10 \%$, respectively, at the same speed $v=0.6 c 0$. 

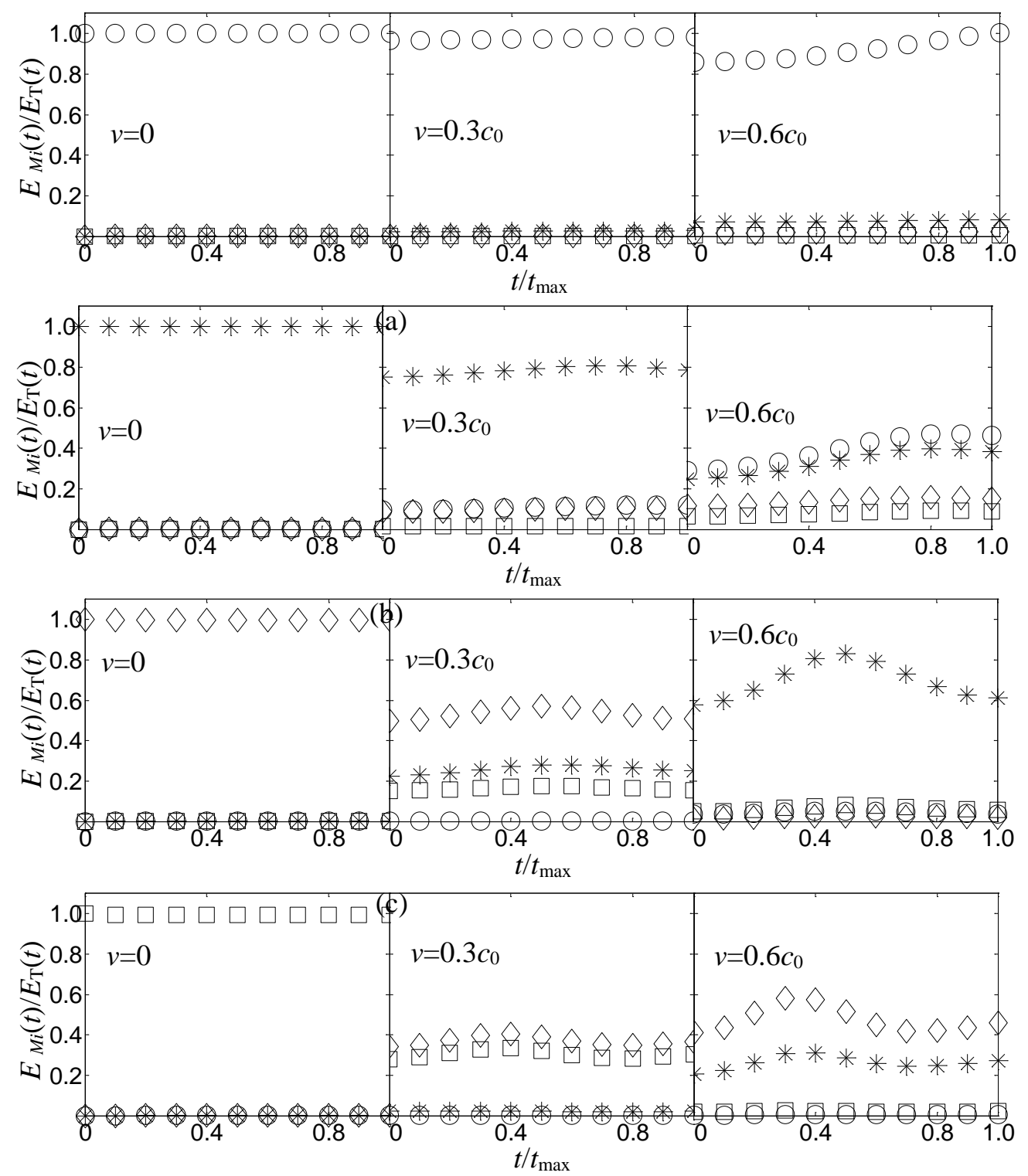

Fig.8 The first four modal energy curves of a string $)_{\text {with }}$ speed of $v=0, v=0.3 c_{0}$ and $v=0.6 c_{0}$, respectively, are identified using ooo the first, $* * *$ the second,$\diamond \diamond \diamond$ the third and $\square \square \square$ the fourth order. The initial displacements are in its (a) first, (b) second, (c) third and (d) fourth order modal shapes, respectively.

\section{Conclusions}

Lagrangian function in conjunction with the finite element method has been applied to develop a transverse vibration equations for a translating string with either constant or variable length. Elements having a quadratic shape function were used and a subsequent nonlinear transform provides a numerical solution for the nonlinear model.

The proposed adaptive TV-DOF technique, applied in the Newmark-Beta method, guarantee equal element lengths automatically when the string length is changing. Other approximate numerical methods are applicable for the case of a small variation in string length, while the proposed method is much superior in accuracy and stability when the length varies greatly.

Using a complex mode theory, the modal components as well as the modal energy for a 
travelling string were expressed analytically. The changing of mode shapes with different $v$ causes the leakage of vibrational energy from one mode to its neighbouring modes. For increasing speed and higher mode order, the phenomenon of energy transfer to other modes is more clearly obvious and significant.

\section{Acknowledgments}

The authors are greatly indebted to the National Natural Science Foundation of China (Grant No.51675150 and 51305115) for the support of this research.

\section{Declaration of conflicting interests}

The authors declared no potential conflicts of interest with respect to the research, authorship, and publication of this article.

\section{References}

Suweken G and Van Horssen WT (2003) On the weakly nonlinear, transversal vibrations of a conveyor belt with a low and time-varying velocity. Journal of Sound and Vibration 267 (5): 1007-1027.

Zhu WD and Xu GY (2003) Vibration of elevator cables with small bending stiffness. Journal of Soundand Vibration 263 (3): 679-699.

Stolte J and Benson RC (1992) Dynamic deflection of paper emerging from a channel. Journal of Vibration and Acoustics. 114(2): 187-193.

Kuiper GL and Metrikine AV (2004) On stability of a clamped-pinned pipe conveying fluid. Heron 49 (3): 211-232.

Kaczmarczyk S and Ostachowicz W (2003) Transient vibration phenomena in deep mine hoisting cables. Part1: Mathematical model. Journal of Sound and Vibration 262 (2): 219-244.

Carrier CF (1945) On the vibration problem of elastic string. Journal of Applied Mathematics 3: 151-165.

Archibald FR, Emslie AG (1958) The vibrations of a string having a uniform motion along its length. Journal of Applied Mechanics-Transactions of the ASME 25: 347-348.

Yao CM, Fung RF and Tseng CR (1999) Non-linear vibration analysis of a travelling string with time-dependent length by new hybrid Laplace transform/finite element method. Journal of Sound and Vibration 219(2): 323-337.

Fung RF, Huang JS, Chu JJ (1998) Dynamic stability of an axially travelling string/slider coupling system with moving boundary. Journal of Sound and Vibration 211 (4): 689-701.

Zhang NH (2008) Dynamic analysis of an axially moving viscoelastic string by the Galerkin method using translating string eigenfunctions. Chaos, Solitons and Fractals 35: 291-302.

Chen LQ, Zhao WJ and Ding H (2009) On Galerkin discretization of axially moving nonlinear strings. Acta Mechanica Solida Sinica, 22(4): 369-376.

Chen EW, Luo Q, Ferguson NS, et al. (2017) A reflected wave superposition method for vibration and energy of a travelling string. Journal of Sound and Vibration, 400C: 40-57.

Sack RA (1954) Transverse oscillations in travelling strings, British Journal of Applied Physics 5 (6):224-226 
Matsuzaki Y, Taki Y and Toyama M (1995) Vibration of a cantilever beam during deployment and retrieval: analysis and experiment, Smart Material and Structures 4: 334-339.

Al-Bedoor BO and Khulief YA (1997) General planar dynamics of a sliding flexible link, Journal of Sound and Vibration 206: 641-661.

Chen EW and Ferguson NS (2014) Analysis of energy dissipation in an elastic moving string with a viscous damper at one end, Journal of sound and vibration. 333 (9): 2556-2570.

Petyt M (2010) Introduction to finite element vibration analysis. Second edition, Cambridge University Press, Ney York, USA.

Li DB, Lu QH (2001) Experimental modal analysis and Its applications, Science Press, China.

Kotera T (1978) Vibrations of string with time-varying length, Bulletin of JSME. 21(160): 1469-1474.

Li QY (2008) Numerical analysis (Fifth edition), Tsinghua University Press, China.

\section{Appendix 1. Detail expression for parameters.}

$$
\begin{aligned}
& \mathbf{k}_{j 1}=\frac{\rho l(t)^{2}}{15 l(t) n}\left[\begin{array}{ccc}
35 j^{2}-55 j+23 & -40 j^{2}+60 j-26 & 5 j^{2}-5 j+3 \\
-40 j^{2}+60 j-26 & 80 j^{2}-80 j+32 & -40 j^{2}+20 j-6 \\
5 j^{2}-5 j+3 & -40 j^{2}+20 j-6 & 35 j^{2}-15 j+3
\end{array}\right], \\
& \mathbf{k}_{j 2}=\frac{\rho \dot{l}(t) \dot{x}(t)}{6 l(t)}\left[\begin{array}{ccc}
-14 j+11 & 16 j-12 & -2 j+1 \\
16 j-12 & -32 j+16 & 16 j-4 \\
-2 j+1 & 16 j-4 & -14 j+3
\end{array}\right], \quad \mathbf{k}_{j 3}=\frac{\left(\rho \dot{x}(t)^{2}-T_{0}\right) n}{3 l(t)}\left[\begin{array}{ccc}
7 & -8 & 1 \\
-8 & 16 & -8 \\
1 & -8 & 7
\end{array}\right], \\
& \mathbf{c}_{j 1}=\frac{\rho \dot{l}(t)}{30 n}\left[\begin{array}{ccc}
15 j-13 & 20 j-14 & -5 j+2 \\
-20 j+16 & 8 & 20 j-4 \\
5 j-3 & -20 j+6 & -15 j+2
\end{array}\right], \mathbf{c}_{j 2}=\frac{\rho \dot{x}(t)}{6}\left[\begin{array}{ccc}
-3 & 4 & -1 \\
-4 & 0 & 4 \\
1 & -4 & 3
\end{array}\right], \mathbf{m}_{j}=\frac{\rho l(t)}{30 n}\left[\begin{array}{ccc}
4 & 2 & -1 \\
2 & 16 & 2 \\
-1 & 2 & 4
\end{array}\right] \text {, } \\
& \mathbf{M}_{\mathrm{L}}(t)=\left[\begin{array}{ccc}
\mathbf{m}_{1} & 0 & 0 \\
0 & \ddots & 0 \\
0 & 0 & \mathbf{m}_{3 n}
\end{array}\right], \quad \mathbf{C}_{\mathrm{L}}(t)=\left[\begin{array}{ccc}
\mathbf{c}_{1} & 0 & 0 \\
0 & \ddots & 0 \\
0 & 0 & \mathbf{c}_{3 n}
\end{array}\right], \quad \mathbf{K}_{\mathrm{L}}(t)=\left[\begin{array}{ccc}
\mathbf{k}_{1} & 0 & 0 \\
0 & \ddots & 0 \\
0 & 0 & \mathbf{k}_{3 n}
\end{array}\right], \quad \mathbf{N}_{\mathrm{L}}(t)=\left[\begin{array}{c}
\hat{\mathbf{S}}_{1} \\
\hat{\mathbf{S}}_{2} \\
\vdots \\
\hat{\mathbf{S}}_{n}
\end{array}\right], \\
& \mathbf{Q}=\left[\begin{array}{lllll}
Q_{1} & Q_{2} & Q_{3} & \cdots & Q_{2 n+1}
\end{array}\right]^{T}, \quad \mathbf{q}=\left[\begin{array}{llll}
\mathbf{q}_{1} & \mathbf{q}_{2} & \cdots & \mathbf{q}_{3 n}
\end{array}\right]^{T}, \\
& \hat{\mathbf{S}}_{j}\left(q_{3 j-2}, q_{3 j-1}, q_{3 j}\right)= \\
& \frac{n^{3}}{10 l(t)^{3}} E A\left[\begin{array}{c}
\left(61 q_{3 j-2}^{3}-216 q_{3 j-2}^{2} q_{3 j-1}+33 q_{3 j-2}^{2} q_{3 j}+272 q_{3 j-2} q_{3 j-1}^{2}-112 q_{3 j-2} q_{3 j-1} q_{3 j}\right. \\
\left.+23 q_{3 j-2} q_{3 j}^{2}-128 q_{3 j-1}^{3}+112 q_{3 j-1}^{2} q_{3 j}-56 q_{3 j-1} q_{3 j}^{2}+11 q_{3 j}^{3}\right) \\
8\left(q_{3 j-2}-2 q_{3 j-1}+q_{3 j}\right)\left(-9 q_{3 j-2}^{3}+16 q_{3 j-2} q_{3 j-1}+2 q_{3 j-2} q_{3 j}-16 q_{3 j-1}^{2}+16 q_{3 j-1} q_{3 j}-9 q_{3 j}^{2}\right)
\end{array}\right], \\
& \left(11 q_{3 j-2}^{3}-56 q_{3 j-2}^{2} q_{3 j-1}+23 q_{3 j-2}^{2} q_{3 j}+112 q_{3 j-2} q_{3 j-1}^{2}-112 q_{3 j-2} q_{3 j-1} q_{3 j}\right. \\
& \left.+33 q_{3 j-2} q_{3 j}^{2}-128 q_{3 j-1}^{3}+272 q_{3 j-1}^{2} q_{3 j}-216 q_{3 j-1} q_{3 j}^{2}+61 q_{3 j}^{3}\right)
\end{aligned}
$$




$$
\mathbf{B}=\left[\begin{array}{cccccccccccc}
1 & 0 & 0 & 0 & 0 & 0 & 0 & \cdots & 0 & 0 & 0 & 0 \\
0 & 1 & 0 & 0 & 0 & 0 & 0 & \cdots & 0 & 0 & 0 & 0 \\
0 & 0 & 1 & 1 & 0 & 0 & 0 & \cdots & 0 & 0 & 0 & 0 \\
0 & 0 & 0 & 0 & 1 & 0 & 0 & \cdots & 0 & 0 & 0 & 0 \\
0 & 0 & 0 & 0 & 0 & 1 & 1 & \cdots & 0 & 0 & 0 & 0 \\
\vdots & \vdots & \vdots & \vdots & \vdots & \vdots & \vdots & \ddots & \vdots & \vdots & \vdots & \vdots \\
0 & 0 & 0 & 0 & 0 & 0 & 0 & \cdots & 1 & 1 & 0 & 0 \\
0 & 0 & 0 & 0 & 0 & 0 & 0 & \cdots & 0 & 0 & 1 & 0 \\
0 & 0 & 0 & 0 & 0 & 0 & 0 & \cdots & 0 & 0 & 0 & 1
\end{array}\right]^{T} \quad \text { Matrix } \mathbf{B} \text { has the size of } 3 n \times(2 n+1)
$$

\section{Appendix 2. Derivation for the quadratic shape function.}

The abscissa for the three nodes in the $j^{\text {th }}$ element can be expressed as

$$
\left\{\begin{aligned}
x_{3 j-2} & =(j-1) l / n \\
x_{3 j-1} & =(2 j-1) l / 2 n \\
x_{3 j} & =j l / n
\end{aligned}\right.
$$

Substitute three coordinate values $\left(x_{3 j-2}, q_{3 j-2}\right),\left(x_{3 j-1}, q_{3 j-1}\right)$ and $\left(x_{3 j}, q_{3 j}\right)$ into a function of quadratic curves

$$
q(x)=a_{1}+a_{2} x+a_{3} x^{2}=\left[1, x, x^{2}\right]\left[a_{1}, a_{2}, a_{3}\right]^{\mathrm{T}}
$$

where, $a_{1}, a_{2}$ and $a_{3}$ are undetermined coefficients, one has

$$
\left[\begin{array}{l}
q_{3 j-2} \\
q_{3 j-1} \\
q_{3 j}
\end{array}\right]=\left[\begin{array}{lll}
1 & x_{3 j-2} & x_{3 j-2}^{2} \\
1 & x_{3 j-1} & x_{3 j-1}^{2} \\
1 & x_{3 j} & x_{3 j}^{2}
\end{array}\right]\left[\begin{array}{l}
a_{1} \\
a_{2} \\
a_{3}
\end{array}\right]
$$

The undetermined coefficients can be obtained

$$
\left[\begin{array}{l}
a_{1} \\
a_{2} \\
a_{3}
\end{array}\right]=\left[\begin{array}{lll}
1 & x_{3 j-2} & x_{3 j-2}^{2} \\
1 & x_{3 j-1} & x_{3 j-1}^{2} \\
1 & x_{3 j} & x_{3 j}^{2}
\end{array}\right]^{-1}\left[\begin{array}{l}
q_{3 j-2} \\
q_{3 j-1} \\
q_{3 j}
\end{array}\right]
$$

Substitute Eq.(d) into (b), one has

$$
q(x)=\left[1, x, x^{2}\right]\left[\begin{array}{lll}
1 & x_{3 j-2} & x_{3 j-2}^{2} \\
1 & x_{3 j-1} & x_{3 j-1}^{2} \\
1 & x_{3 j} & x_{3 j}^{2}
\end{array}\right]^{-1}\left[\begin{array}{l}
q_{3 j-2} \\
q_{3 j-1} \\
q_{3 j}
\end{array}\right]
$$

Define the quadratic shape function as follows

$$
\mathbf{N}_{j}(x, l)=\left[1, x, x^{2}\right]\left[\begin{array}{lll}
1 & x_{3 j-2} & x_{3 j-2}^{2} \\
1 & x_{3 j-1} & x_{3 j-1}^{2} \\
1 & x_{3 j} & x_{3 j}^{2}
\end{array}\right]^{-1}
$$

and substitute Eq.(a) into (f), one can obtain the detailed expression for $\mathbf{N}_{j}(x, l(t))$.

$$
\mathbf{N}_{j}=\left[-\frac{(j l(t)-n x)(l(t)-2 j l(t)+2 n x)}{l(t)^{2}}, \frac{4(j l(t)-n x)(l(t)-j l(t)+n x)}{l(t)^{2}}, \frac{(l(t)-j l(t)+n x)(l(t)-2 j l(t)+2 n x)}{l(t)^{2}}\right]
$$

